\title{
Comparison of Psychological Morbidity of Health Care Workers Posted in COVID and Non COVID Labour Rooms
}

\author{
Archana Mishra ${ }^{1} \cdot$ Sheeba Marwah $^{1} \cdot$ Renu Arora $^{1} \cdot$ Abhilasha Yadav $^{2} \cdot$ Nupur Anand $^{1} \cdot$ Dalimi Mushahary $^{1}$
}

Received: 31 January 2021 / Accepted: 8 February 2021 / Published online: 2 April 2021

(c) Federation of Obstetric \& Gynecological Societies of India 2021

\begin{abstract}
Background The COVID-19 pandemic had not only burdened healthcare systems all over the world but had also affected mental health of frontline Health Care Workers.

Purpose Purpose of our study was to compare the psychological morbidity of health care workers posted in Covid labour room with their counter parts in Non Covid labour room.

Material and Methods Present study was a cross-sectional study conducted on HCW of Covid and Non Covid labour in a span of 6 months.

Results Insomnia in Covid labour room was found to be in $57 \% \mathrm{HCW}$ as compared to none in non Covid labour room (mean score $7.47 \pm 2.74$ vs $1.82 \pm 1.25, P$ Value $<.0001)$. Depression was prevalent in $92 \%$ of participants in Covid labour room as compared to $12.5 \%$ in Non Covid labour room; however, severe depression was found in $6 \%$ participants (mean score $17.32 \pm 4.88$ vs $2.12 \pm 1.65, P$ Value $<.0001$ ). Prevalence of anxiety in present study was $90 \%$ in Covid labour room and $6 \%$ in non Covid labour room (mean score $11.47 \pm 4.57$ vs $1.94 \pm 1.43, P$ Value $<.0001$ ). Psychological morbidity as tested by Symptom Check List-k-9 was positive in $21.8 \%$ participants in Covid Labour room as compared to $1 \%$ in Non Covid labour room (mean score $5.57 \pm 2.58$ vs $2.22 \pm 1.89, P$ Value $<.0001$ )

Conclusion Study revealed significantly high psychological morbidity, insomnia, anxiety and depression in the health care workers in Covid labour room than Non Covid labour room.
\end{abstract}

Keywords Psychological morbidity $\cdot$ Health care workers $\cdot$ Covid labour room $\cdot$ Anxiety $\cdot$ Depression $\cdot$ Insomnia

Archana Mishra is an Associate Professor in Obstetrics and Gynaecology, Vardhman Mahavir Medical College and Safdarjung Hospital, New Delhi, Delhi, India; Sheeba Marwah is an Assistant Professor in Obstetrics and Gynaecology, Vardhman Mahavir Medical College and Safdarjung Hospital, New Delhi, Delhi, India; Renu Arora is a Professor in Obstetrics and Gynaecology, Vardhman Mahavir Medical College and Safdarjung Hospital, New Delhi, Delhi, India; Abhilasha Yadav is a Professor, Psychiatry, Vardhman Mahavir Medical College and Safdarjung Hospital, New Delhi, Delhi, India; Nupur Anand is a Senior Resident in Obstetrics and Gynaecology, Vardhman Mahavir Medical College and Safdarjung Hospital, New Delhi, Delhi, India; Dalimi Mushahary is a Post Graduate in Obstetrics and Gynaecology, Vardhman Mahavir Medical College and Safdarjung Hospital, New Delhi, Delhi, India.

Archana Mishra

drarchanamishra@rediffmail.com

1 Obstetrics and Gynaecology, Vardhman Mahavir Medical College and Safdarjung Hospital, New Delhi, Delhi, India

2 Vardhman Mahavir Medical College and Safdarjung Hospital, New Delhi, Delhi, India

$\begin{array}{ll}\text { Abbreviations } & \\ \text { COVID } & \begin{array}{l}\text { Corona Virus Disease } \\ \text { Health Care Worker } \\ \text { HCW }\end{array} \\ \text { SARS } & \begin{array}{l}\text { Severe Acute Respiratory } \\ \text { Syndrome }\end{array} \\ \text { SCL K-9 } & \text { Symptom Check List K-9 } \\ \text { ISI } & \text { Insomnia Severity Index } \\ \text { PHQ-9 } & \begin{array}{l}\text { Patient Health Questionnaire } \\ \text { 9-item }\end{array} \\ \text { GAD-7 } & \text { Generalized Anxiety Disorder } \\ \text { Covid Labour room } & \text { Labour room for delivery of } \\ & \text { covid positive women }\end{array}$

Non Covid Labour room Labour room for delivery of all women except known case of covid positive 


\section{Introduction:}

World health organization designated COVID-19 [SARSCoV2] outbreak a public health emergency in January 2020 which later on was declared as a pandemic in the month of March same year [1]. COVID-19 pandemic has put health care workers all over the world in an unprecedented situation. A few researchers had addressed the psychological impact of pandemic over health care workers [2]. Most of the studies are only from one country that is China. There is paucity of literature from other countries. Most of the studies have focused on anaesthesia and medicine specialty only and very less data is available from obstetrics. Working in labour room is psychologically demanding due to need of immediate attention, quick crucial decisions regarding maternal and fetal wellbeing and emergency situations like postpartum haemorrhage. Obstetricians and labour room staff posted in covid labour room face more challenges due to prolonged direct exposure during monitoring of labour and delivery, exposure to potentially fatal contamination, physical exhaustion due to long working hours with personal protective equipment and working in an unadjusted environment. During pandemic our labour room workforce was divided in two halves to cater to covid labour room and non covid labour room. Purpose of our study was to evaluate the psychological morbidity of Health care workers posted in covid labour room and compare it with their counterparts posted in non covid labour room. Our study also evaluated the reasons contributing to higher psychological morbidity in the staff posted in covid labour room.

\section{Methods}

Present study was a cross-sectional study conducted in the department of Obstetrics and Gynaecology of a tertiary care centre of New Delhi in a span of 6 months from August 2020 to January 2021. Institutional ethics committee approved the study [IEC/VMMC/SJH/Projects/2020-08/CC-5].

\section{Inclusion Criteria}

Health care workers posted in covid labour room and non covid labour room including doctors, nurses and other frontline healthcare workers.

\section{Exclusion Criteria}

1. Diagnosed psychiatric illness.
2. Medications affecting psychological wellbeing.

3. HCW not directly involved in patient care.

$\mathrm{HCW}$ including doctors,nursing staff and other frontline health care workers posted inside the labour rooms were contacted and informed in detail about the study. After recruitment $\mathrm{HCW}$ were asked to self-administer the prestructured proforma in the middle of their posting in their respective labour rooms. Confidentiality of the data was maintained. HCW undergoing psychological stress were offered telephonic/on site counselling by a co-investigator from psychiatry department.

\section{Measurement Tools}

Using the questionnaire, we collected demographic data and administered the SCL-K 9 for severity of global symptoms of psychological distress, insomnia scale, depressive symptom scale and anxiety symptom scale.

SCL-K-9 was used to measure psychological distress of participants. A total score more than or equal to 7 was taken as positive [3].

The Insomnia Severity Index (ISI) was used to measure the severity of insomnia. Each item was rated on a 0-4 scale, and the total score ranged from 0 to 28 . Higher score suggested more severe insomnia symptoms. A total score of $\geq 8$ was considered as having symptoms of insomnia.

The Patient Health Questionnaire 9-item depression module (PHQ-9) was used to measure depressive symptoms. Each of the nine items were scored on a scale from 0 to 3 . The total score suggested different levels of depressive symptoms: minimal/no depression (0-4), mild depression (5-9), moderate depression (10-14) or severe depression (15-21).

The Generalized Anxiety Disorder (GAD-7) scale was used to assess anxiety. The total score can be categorized into four severity groups: minimal/no anxiety (0-4), mild anxiety (5-9), moderate anxiety (10-14) or severe anxiety (15-21).

\section{Statistical Analysis:}

The presentation of the Categorical variables was done in the form of number and percentage (\%). On the other hand, the presentation of the continuous variables was done as mean \pm SD and median values. The data normality was checked by using Kolmogorov-Smirnov test. The cases in which the data were not normal, we used non parametric 
tests. The following statistical tests were applied for the results:

1. The comparison of the variables which were quantitative in nature were analyzed using Mann-Whitney Test (for two groups).

2. The comparison of the variables which were qualitative in nature were analyzed using Chi Square test/Fisher's Exact test.

The data entry was done in the Microsoft EXCEL spreadsheet and the final analysis was done with the use of Statistical Package for Social Sciences (SPSS) software version 21.0.

For statistical significance, $\mathrm{p}$ value of less than 0.05 was considered as significant.

\section{Results}

Results were available for 400 participants, 200 in each group of covid labour room and non covid labour room. All our participants were females. Table 1 reveals base line characteristics of participants. Table 2 describes comparison of social characteristics between covid and non covid labour room. Table 3 compares psychological morbidity scores between covid and non covid labour room. Table 4 shows comparison of perceived stressors between covid and non covid labour room.

Insomnia was found to be in $57 \%$ of health care workers in covid labour room as compared to none in non covid labour room. Depression was prevalent in $92 \%$ of participants in covid labour room as compared to $12.5 \%$ in non covid labour room; however, severe depression was found only in $6 \%$ participants. Prevalence of anxiety in present study was $90 \%$ in $\mathrm{HCW}$ in covid labour room and $6 \%$ in $\mathrm{HCW}$ in non covid labour room. Psychological morbidity as tested by Symptom Check List -9 was positive in $21.8 \%$ participants in covid labour room as compared to $1 \%$ in non covid labour room.

\section{Discussion}

Present study is one of the very few studies which assessed and compared psychological morbidity (anxiety, insomnia, depression and symptoms of psychological distress) in age, sex and profession matched healthcare workers posted in covid versus non covid labour room. There are many more

Table 1 Comparison of baseline characteristics of HCW in covid and non covid labour room

\begin{tabular}{|c|c|c|c|c|c|}
\hline Characteristics & $\begin{array}{l}\text { Covid } \\
(n=200)\end{array}$ & $\begin{array}{l}\text { Non covid } \\
(n=200)\end{array}$ & Total & $P$ value & $\begin{array}{l}\text { Test } \\
\text { performed }\end{array}$ \\
\hline \multicolumn{6}{|l|}{ Age(years) } \\
\hline Mean \pm SD & $28.23 \pm 5.73$ & $27.64 \pm 4.22$ & $27.94 \pm 5.04$ & 0.461 & Mann Whitney test; $19,159.5$ \\
\hline Median(25th-75th percentile) & $27(25-28)$ & $27(26-28)$ & $27(26-28)$ & & \\
\hline Range & $24-55$ & $24-53$ & $24-55$ & & \\
\hline \multicolumn{6}{|l|}{ Marital status } \\
\hline Married & $20(10 \%)$ & $120(60 \%)$ & $136(34 \%)$ & $<.0001$ & Chi square test, 109.89 \\
\hline Unmarried & $180(90 \%)$ & $80(40 \%)$ & $260(65 \%)$ & & \\
\hline \multicolumn{6}{|l|}{ Designation } \\
\hline Intern & $32(16 \%)$ & $32(16 \%)$ & $64(32 \%)$ & 0.649 & Fisher Exact test \\
\hline Nurse & $56(28 \%)$ & $66(33 \%)$ & $122(30.50 \%)$ & & \\
\hline Nursing orderly & $12(6 \%)$ & $14(7 \%)$ & $26(6.5 \%)$ & & \\
\hline Postgraduate Resident & $54(27 \%)$ & $42(21 \%)$ & $96(24 \%)$ & & \\
\hline Senior resident & $46(23 \%)$ & $46(23 \%)$ & $92(23 \%)$ & & \\
\hline \multicolumn{6}{|l|}{ Experience(years) } \\
\hline $0-5$ years & $100(50 \%)$ & $92(46 \%)$ & $192(48 \%)$ & 0.04 & Fisher Exact test \\
\hline $5-10$ years & $100(50 \%)$ & $102(51 \%)$ & $202(50.50 \%)$ & & \\
\hline $10-15$ years & $0(0 \%)$ & $6(3 \%)$ & $6(1.50 \%)$ & & \\
\hline \multicolumn{6}{|l|}{ Co-morbidity } \\
\hline No & $198(99 \%)$ & $172(86 \%)$ & $370(92.50 \%)$ & $<.0001$ & Fisher Exact test \\
\hline Yes & $2(1 \%)$ & $28(14 \%)$ & $30(7.50 \%)$ & & \\
\hline
\end{tabular}

There was no significant difference in age group,designation and experience of the participants of both labour rooms however co-morbidities were present significantly higher in participants of non covid labour room and there were more unmarried participants in covid labour room 
Table 2 Comparison of social characteristics between HCW of covid and non covid labour room

\begin{tabular}{|c|c|c|c|c|c|}
\hline Social characteristics & $\begin{array}{l}\text { Covid } \\
(n=200)\end{array}$ & $\begin{array}{l}\text { Non covid } \\
(n=200)\end{array}$ & Total & $P$ value & $\begin{array}{l}\text { Test } \\
\text { performed }\end{array}$ \\
\hline Living alone & $130(65 \%)$ & $60(30 \%)$ & $190(47.5 \%)$ & $<.0001$ & Chi square test, 92.754 \\
\hline Children in family & $70(35 \%)$ & $82(41 \%)$ & $152(38 \%)$ & 0.216 & Chi square test, 1.528 \\
\hline Elderly in family & $52(26 \%)$ & $59(29.50 \%)$ & $111(27.75 \%)$ & 0.434 & Chi square test, 0.611 \\
\hline Single earning member & $9(4.50 \%)$ & $20(10 \%)$ & $29(7.25 \%)$ & 0.034 & Chi square test, 4.499 \\
\hline Opted for quarantine at home & $163(81.50 \%)$ & $162(81 \%)$ & $325(81.25 \%)$ & 0.898 & Chi square test, 0.016 \\
\hline Using public transport & $134(67 \%)$ & $59(29.50 \%)$ & $193(48.25 \%)$ & $<.0001$ & Chi square test, 56.319 \\
\hline $\begin{array}{l}\text { Frequently communicate with } \\
\text { friends/family }\end{array}$ & $93(46.50 \%)$ & $141(70.50 \%)$ & $234(58.50 \%)$ & $<.0001$ & Chi square test, 23.726 \\
\hline
\end{tabular}

Significantly more number of participants in covid labour room were living alone and using public transport. Non covid labour room had significantly higher number of participants who were single earning members and they communicated more frequently with family and friends during labour room posting

Table 3 Comparison of psychological morbidity scores of HCW between covid and non covid labour room

\begin{tabular}{|c|c|c|c|c|c|}
\hline Psychological morbidity scores & $\begin{array}{l}\text { Covid } \\
(n=200)\end{array}$ & $\begin{array}{l}\text { Non Covid } \\
(n=200)\end{array}$ & Total & $P$ value & $\begin{array}{l}\text { Test } \\
\text { performed }\end{array}$ \\
\hline \multicolumn{6}{|l|}{$S C L K-9$} \\
\hline$<=7$ & $157(78.50 \%)$ & $198(99 \%)$ & $355(88.75 \%)$ & $<.0001$ & Fisher Exact test \\
\hline$>7$ & $43(21.50 \%)$ & $2(1 \%)$ & $45(11.25 \%)$ & & \\
\hline Mean \pm SD & $5.57 \pm 2.58$ & $2.22 \pm 1.89$ & $3.9 \pm 2.81$ & $<.0001$ & Mann Whitney test;5676 \\
\hline Median(25th-75th percentile) & $6(4-7)$ & $2(1-3)$ & $4(2-6)$ & & \\
\hline Range & $0-19$ & $0-8$ & $0-19$ & & \\
\hline \multicolumn{6}{|l|}{ GAD 7 scale } \\
\hline No anxiety & $16(8 \%)$ & $188(94 \%)$ & $204(51 \%)$ & $<.0001$ & Chi square test,295.958 \\
\hline Anxiety & $180(90 \%)$ & $12(6 \%)$ & $192(48 \%)$ & & \\
\hline Mean \pm SD & $11.47 \pm 4.57$ & $1.94 \pm 1.43$ & $6.71 \pm 5.84$ & $<.0001$ & Mann Whitney test;637 \\
\hline Median(25th-75th percentile) & $11(9-15)$ & $2(1-3)$ & $4(2-11)$ & & \\
\hline Range & $3-20$ & $0-6$ & $0-20$ & & \\
\hline \multicolumn{6}{|l|}{$P H Q 9$} \\
\hline No depression & $16(8 \%)$ & $175(87.50 \%)$ & $175(43.75 \%)$ & $<.0001$ & Fisher Exact test \\
\hline Depression & $184(92 \%)$ & $25(12.50 \%)$ & $225(56.25 \%)$ & & \\
\hline Mean \pm SD & $17.32 \pm 4.88$ & $2.12 \pm 1.65$ & $9.72 \pm 8.44$ & $<.0001$ & Mann Whitney test;0 \\
\hline Median(25th-75th percentile) & $18(13-22)$ & $2(1-3)$ & $6.5(2-18)$ & & \\
\hline Range & $7-26$ & $0-6$ & $0-26$ & & \\
\hline \multicolumn{6}{|l|}{ Insomnia severity index } \\
\hline No insomnia & $86(43 \%)$ & $200(100 \%)$ & $286(71.50 \%)$ & $<.0001$ & Fisher Exact test \\
\hline Insomnia & $114(57 \%)$ & $0(0 \%)$ & $114(28.50 \%)$ & & \\
\hline Mean \pm SD & $7.47 \pm 2.74$ & $1.82 \pm 1.25$ & $4.64 \pm 3.54$ & $<.0001$ & Mann Whitney test; 1870 \\
\hline Median(25th-75th percentile) & $8(6-9)$ & $2(1-2)$ & $4(2-8)$ & & \\
\hline Range & $0-12$ & $0-5$ & $0-12$ & & \\
\hline
\end{tabular}

Scores of SCL K 9, Insomnia severity index, PHQ-9, and GAD -7 all are significantly higher in health care workers of covid labour room as compared to non covid labour room

studies on psychological aspect of HCW but direct comparison with other studies should be made with caution due to difference in assessment tools, survey methods, sociocultural difference and different working environments.
Prevalence of insomnia in health care workers in covid labour room was found to be 57\% as compared to none in non covid labour room which was significantly high.Pandemic-related stress induces psychological and physical activation in response. This activated 
Table 4 Comparison of perceived stressors between covid and non covid labour room

\begin{tabular}{|c|c|c|c|c|c|}
\hline Perceived stressors & $\begin{array}{l}\text { Covid } \\
(n=200)\end{array}$ & Non covid $(n=200)$ & Total & $P$ value & $\begin{array}{l}\text { Test } \\
\text { performed }\end{array}$ \\
\hline Lack of desired facility for self and for patients & $12(6 \%)$ & $23(11.50 \%)$ & $35(8.75 \%)$ & 0.052 & Chi square test, 3.789 \\
\hline Inadequate man power & $10(5 \%)$ & $23(11.50 \%)$ & $33(8.25 \%)$ & 0.018 & Chi square test, 5.582 \\
\hline Fear of infection to self & $120(60 \%)$ & $76(38 \%)$ & $196(49 \%)$ & $<.0001$ & Chi square test, 19.368 \\
\hline Fear of infecting family & $117(58.50 \%)$ & $80(40 \%)$ & $197(49.25 \%)$ & 0.0002 & Chi square test, 13.693 \\
\hline Unsure future of pandemic & $15(7.50 \%)$ & $6(3 \%)$ & $21(5.25 \%)$ & 0.044 & Chi square test, 4.071 \\
\hline $\begin{array}{l}\text { Overload of information and frequent change of guide- } \\
\text { lines }\end{array}$ & $10(5 \%)$ & $6(3 \%)$ & $16(4 \%)$ & 0.307 & Chi square test, 1.042 \\
\hline Exhaustion & $32(16 \%)$ & $28(14 \%)$ & $60(15 \%)$ & 0.575 & Chi square test, 0.314 \\
\hline Guilt of not taking proper care of family & $2(1 \%)$ & $0(0 \%)$ & $2(0.50 \%)$ & 0.499 & Fisher Exact test \\
\hline Social Stigma & $1(0.50 \%)$ & $0(0 \%)$ & $1(0.25 \%)$ & 1 & Fisher Exact test \\
\hline
\end{tabular}

Most significantly associated stress factors were "risk of infection to self" followed by "worries about infecting the friends and family members". Other important factors were "unsure future of pandemic". Non covid labour room HCW were significantly stressed with inadequate manpower to deal with high number of patients and long working hours

hypothalamus-pituitary-adrenal (HPA) system is not compatible with normal sleep which ultimately forms a vicious cycle resulting in sleep disorders. Other studies from China and Italy revealed the prevalence of insomnia as $36.1 \%$ and $8.27 \%$ during covid pandemic $[4,5]$. Study from Italy revealed that being female and younger age were associated with higher insomnia severity.

In present study, depression as assessed by PHQ-9 was prevalent in $92 \%$ of participants in covid labour room as compared to $12.5 \%$ in non covid labour room.In present study, however, all participant were of similar age and profession but number of healthcare workers living alone was much higher in covid labour room which may be the reason for high prevalence of depression.

Loneliness as a reason for high PHQ-9 has been quoted by another scholar who reported prevalence of depression as $43.6 \%$ in nurses of emergency department during covid pandemic [6]. Similar findings were suggested by two other researchers from China which showed prevalence of depression in frontline clinicians as $54.4 \%$ and $31.37 \%$ [7, 8].

Prevalence of anxiety in present study was $90 \%$ in HCW in covid labour room and 6\% in $\mathrm{HCW}$ in non covid labour room. However, another study recorded a $9.4 \%$ prevalence of anxiety in nurses in pandemic [9].

Symptom Check List-9 for psychological morbidity was used in present study and found $21.8 \%$ morbidity in participants in covid labour room as compared to $1 \%$ in non covid labour room. Symptom Check List -90 had been administered by one study and found a $11.2 \%$ prevalence of psychological morbidity in health care workers in covid pandemic [10].

Anxiety, depression and insomnia were significantly higher in participants of covid labour room and the prevalence was higher than other studies performed in this subject. It may be explained partly by factors like "female sex" and "living alone". However, most of the other studies on this aspect of pandemic were online surveys which included a wide variety of health care workers who were not directly dealing with covid positive patients like pharmacists and nursing students and majority of $\mathrm{HCW}$ were from provinces not affected by COVID-19, while participants in present study were in close contact with covid positive patients for long hours which increased their stress.

We recommend a multicentre study with larger sample size in this aspect of mental health of HCW.

\section{Conclusion}

Present study revealed significantly high psychological morbidity, insomnia, anxiety and depression in the health care workers in covid labour room than non covid labour room. We recommend guidelines to balance work hours for frontline health care staff and strengthening of infrastructure to provide health care worker a healthy and safe environment. Pre-Posting orientation classes are necessary for preparedness of staff for physical and psychological challenges faced in covid labour room. On site and teleconsultation services for psychological support are helpful to improve resilience of healthcare workers in the face of crisis.

Supplementary Information The online version contains supplementary material available at https://doi.org/10.1007/s13224-021-01465-2.

Funding No funding had been received from any agency in this research. 


\section{Compliance with Ethical Standards}

Conflict of interest There are no potential conflicts of interest in this article.

Ethical standards Study have been approved by the appropriate institutional and/or national research ethics committee and have been performed in accordance with the ethical standards as laid down in the 1964 Declaration of Helsinki and its later amendments or comparable ethical standards.

Human or Animal Rights Our research involved human participants. Our study didnot include animals.

Informed consent Proper informed consent was taken from all the participants.

\section{References}

1. Statement on the Second Meeting of the International Health Regulations (2005) Emergency committee regarding the outbreak of novel coronavirus (2019-nCoV). World Health Organization. Director- General's remarks at the media briefing on 2019-nCoV on 11 February 2020. https://www.who.int/dg/speeches/detail/ who-director-general-s-remarks-at-the-media-briefing-on-2019ncov-on-11-february-2020.

2. Yao H, Chen J, Xu Y, et al. Rethinking online mental health services in China during the COVID-19 epidemic. Asian J Psychiatry. 2020. https://doi.org/10.1016/j.ajp.2020.102015.

3. Petrowski K, Schmalbach B, Kliem S, et al. Symptom-ChecklistK-9: Norm values and factorial structure in a representative German sample. PLoS ONE. 2019;14(4):e0213490. https://doi.org/ 10.1371/journal.pone.0213490.

4. Zhang C, Yang L, Liu S, et al. Survey of insomnia and related social psychological factors among medical staff involved in the 2019 novel coronavirus disease outbreak. Front Psychiatr. 2020;11:306. https://doi.org/10.3389/fpsyt.2020.00306.

5. Rossi R, Socci V, Pacitti F, et al. Mental health outcomes among frontline and second-line health care workers during the coronavirus disease 2019 (COVID-19) pandemic in Italy. JAMA Netw Open. 2020;3(5):e2010185. https://doi.org/10.1001/jamanetwor kopen.
6. An Y, Yang Y, Wang A, et al. Prevalence of depression and its impact on quality of life among frontline nurses in emergency during the COVID-19 outbreak. J Affect Disord. 2020;276:312-5. https://doi.org/10.1016/j.jad.2020.06.047.

7. Lai J, Ma S, Wang Y, et al. Factors associated with mental health outcomes among health care workers exposed to coronavirus disease 2019. JAMA Netw Open. 2020. https://doi.org/10.1001/ jamanetworkopen.2020.3976.

8. Zheng CM, Shen F, Tian GQ, Zhang L, et al. Investigation on the stress level and depression of medical staff during the new coronavirus pneumonia outbreak. Zhejiang Med. 2020;42:406-14. https://doi.org/10.1016/j.jad.2020.06.047 (in Chinese).

9. Hong S, Ai M, Xu X, et al. Immediate psychological impact on nurses working at 42 government-designated hospital during COVID-19 outbreak in China: a cross-sectional study. Nurs Outlook. 2021;69(1):6-12. https://doi.org/10.1016/j.outlook.2020.07. 007.

10. Cai W, Lian B, Song X. A cross-sectional study on mental health among health care workers during the outbreak of corona virus disease 2019. Asian J Psychiatr. 2020;51:102111. https://doi.org/ 10.1016/j.ajp.2020.102111.

Publisher's Note Springer Nature remains neutral with regard to jurisdictional claims in published maps and institutional affiliations.

\section{About the Author}

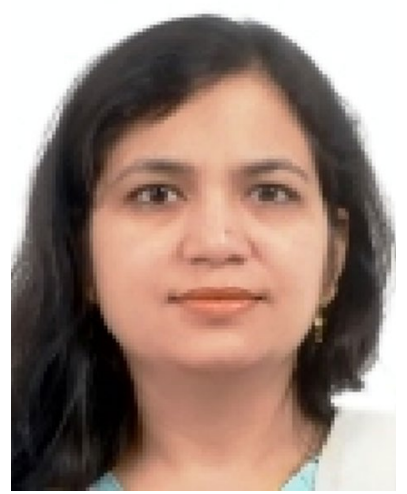

Dr. Archana Mishra is an Associate Professor in Vardhman Mahavir Medical College and Safdarjung Hospital, New Delhi. Her fields of interests are GynaeOncology and minimally invasive surgery including robotic surgery. She has many awards and publications to her credit. At present she is Organizing Secretary of prestigious conference IFCPC 2021. 